\title{
Angiogenic and lymphangiogenic microvessel density in breast carcinoma: correlation with clinicopathologic parameters and VEGF-family gene expression
}

\author{
William WL Choi ${ }^{1}$, Melinda M Lewis ${ }^{1}$, Diane Lawson ${ }^{1}$, Qiqin Yin-Goen ${ }^{1,2}$, \\ George G Birdsong ${ }^{1}$, George A Cotsonis ${ }^{3}$, Cynthia Cohen ${ }^{1}$ and Andrew N Young ${ }^{1,2}$ \\ ${ }^{1}$ Department of Pathology \& Laboratory Medicine, Emory University School of Medicine, Atlanta, GA, USA; \\ ${ }^{2}$ Department of Pathology \& Laboratory Medicine, Atlanta Veterans Affairs Medical Center, Decatur, GA, USA \\ and ${ }^{3}$ Division of Biostatistics, Rollins School of Public Health, Emory University School of Medicine, Atlanta,
} GA, USA

\begin{abstract}
Angiogenesis and lymphangiogenesis are essential for breast cancer progression and are regulated by vascular endothelial growth factors (VEGF). To determine clinical and molecular correlates of these processes, we measured blood and lymphatic vascular microvessel density in 29 invasive carcinomas (22 ductal, six lobular, one papillary), using the vascular marker CD31 and the novel lymphatic marker D2-40. Microvessel density was assessed microscopically and by image cytometry, and was compared with tumor histology, grade, stage, lymph node metastasis, hormone receptors, HER2/neu status, and expression of VEGF, VEGF-C and VEGF-D by immunohistochemistry or quantitative RT-PCR. Strong correlation was observed between visual and image cytometric microvessel density using D2-40 but not CD31 $(P=0.016$ and 0.1521 , respectively). Image cytometric CD31 microvessel density correlated with tumor size, grade, stage and lymph node metastasis $(P=0.0001,0.0107,0.0035$ and 0.0395 , respectively). D2-40 microvessel density correlated with tumor stage $(P=0.0123$ by image cytometry) and lymph node metastasis $(P=0.0558$ by microscopy). Immunohistochemical VEGF signal in peritumoral blood vessels correlated with image cytometric CD31 and D2-40 microvessel density $(P=0.022$ and 0.0012 , respectively), consistent with the role of VEGF in blood and lymphatic vascular growth. Intratumoral VEGF-C and VEGF-D expression by quantitative RT-PCR correlated with D2-40 $(P=0.0291$ by image cytometry) but not with CD31 microvessel density, which could suggest a selective role of VEGF-C and VEGF-D in lymphangiogenesis. CD31 and D2-40 microvessel density correlated significantly with several prognostic factors, including lymph node metastasis. Thus, measurements of angiogenesis and lymphangiogenesis may have utility for breast cancer pathology, particularly for estimation of metastatic risk.
\end{abstract}

Modern Pathology (2005) 18, 143-152, advance online publication, 6 August 2004; doi:10.1038/modpathol.3800253

Keywords: angiogenesis; lymphangiogenesis; immunohistochemistry; image cytometry; quantitative reverse transcriptase-polymerase chain reaction

Angiogenesis (blood vessel growth) and lymphangiogenesis (lymph vessel growth) are critical processes for tumor growth, invasion and metastasis. ${ }^{1-3}$ Thus, measurement of vascular growth may be

Correspondence: Dr AN Young, MD, PhD, Department of Pathology \& Laboratory Medicine, Emory University, Atlanta VA Medical Center, 1670 Clairmont Road, Decatur, GA 30033, USA.

E-mail: Andrew.Young@med.va.gov

Presented at the 93rd Annual Meeting of the United States and Canadian Academy of Pathology, Vancouver, BC, Canada, March 2004.

Received 23 June 2004; revised and accepted 9 July 2004; published online 6 August 2004 clinically important in breast cancer specimens. The common pathologic approach to assessing angiogenesis and lymphangiogenesis involves microscopic estimation of vascular density or microvessel density on tissues probed for endothelial markers by immunohistochemistry. Several markers of blood vessel endothelium have been developed for routine use, including CD31/PECAM-1, CD34, and Factor VIII-related antigen (von Willebrand Factor or vWF). ${ }^{4}$ However, lymphangiogenesis studied have been limited by lack of specific lymphatic endothelial markers. Recently, monoclonal antibody D2-40 was shown in breast ${ }^{5}$ and tonsillar ${ }^{6}$ tissue to detect lymphatic vessels selectively. 
In these studies, lymphatic vessels were immunoreactive for D2-40, while, in contrast, blood vessels were reactive for CD31, CD34, vWF and PAL-E., ${ }^{5,6}$

Studies of various tumors have shown the potential clinical significance of angiogenesis and lymphangiogenesis, suggesting that blood and lymphatic microvessel density correlate with tumor growth and metastasis. ${ }^{7,8}$ These promising results have launched subsequent research aimed at optimizing methods for microvessel density measurement. The two principal approaches are direct microscopic immunohistochemistry and semiautomated image cytometry. Direct immunohistochemical microvessel density is relatively inexpensive and widely available in diagnostic pathology departments, but is subjective and difficult to standardize within and between laboratories. In contrast, image cytometry is more conducive to standardized quantification but is relatively expensive.

In addition to quantification, interest is growing in the molecular regulation of angiogenesis and lymphangiogenesis. Breast cancers elaborate several angiogenic and lymphangiogenic growth factors, which represent candidate markers for diagnosis, prognosis and therapeutic strategies. For example, vascular endothelial growth factor (VEGF) appears to be critical primarily for blood vessel development in breast cancer, while the related factors VEGF-C and VEGF-D regulate lymphatic development. ${ }^{9,10}$ VEGF-C mRNA and protein localize to tumor cells of breast carcinoma, squamous cell carcinoma, lymphoma, melanoma and sarcoma. ${ }^{11}$ VEGFR-3 has been shown to have a role in adult blood vessel angiogenesis. ${ }^{12}$

Previous studies in breast cancer suggest that VEGF-family gene expression correlates with tumor growth and metastasis.9,13 However, it is still unresolved whether VEGF, VEGF-C or VEGF-D expression can be related to blood or lymphatic microvessel density in clinical breast cancer specimens. Therefore, we sought to address this issue by using image cytometry to determine blood or lymphatic microvessel density on a cohort of wellannotated breast carcinoma samples stained with CD31 and D2-40. We analyzed these data together with VEGF, VEGF-C and VEGF-D expression using immunohistochemistry and quantitative RT-PCR.

\section{Materials and methods}

\section{Clinicopathologic Data}

The breast carcinoma samples were obtained from the archives of the Surgical Pathology Division of the Department of Pathology and Laboratory Medicine, Emory University Hospital and Veterans Affairs Medical Center, Atlanta, GA, USA. Cases were diagnosed between 1995 and 2000, and all were deidentified prior to the study. All tumors were invasive carcinomas from female patients. Tumors were subtyped as ductal or lobular carcino- ma, ${ }^{14}$ and histologically graded as I-III based on tubule formation, nuclear grade and mitotic count. ${ }^{15,16}$ Pathologic staging T1-T4 was according to the American Joint Cancer Committee (AJCC) TNM Staging 2002. Regional lymph node, estrogen receptor, progesterone receptor and Her2/neu status were recorded. Pathologic parameters were obtained by review of hematoxylin and eosin (H\&E)-stained slides, surgical pathology reports, and the Oncology Data Bank. This research was approved by the Emory University Institutional Review Board under protocol 015-2001.

\section{Immunohistochemistry}

All tissues were fixed in 10\% neutral buffered formalin and embedded in paraffin using standard surgical pathology protocols. Diagnoses were established from H\&E-stained slides using standard histopathologic criteria. Immunohistochemistry was performed on a single representative block from each case. Tissue sections ( $5 \mu \mathrm{m})$ were dewaxed and antigen retrieval was performed in citrate buffer $(\mathrm{pH}$ 6 ), using an electric pressure cooker set at $120^{\circ} \mathrm{C}$ for 5 min. ${ }^{17}$ Sections were incubated for $5 \mathrm{~min}$ in $3 \%$ hydrogen peroxide to quench endogenous tissue peroxidase. Primary monoclonal antibodies were directed against CD31 (1:80 dilution, DAKO Corporation, Carpinteria, CA, USA), D2-40 antigen (1:5 dilution, Signet Laboratories, Dedham, MA, USA), and VEGF (1:160 dilution, Santa Cruz Biotechnology, Santa Cruz, CA, USA). Tissue sections were incubated with appropriate primary antibody for $25 \mathrm{~min}$ at room temperature. After washing unbound primary antibody, sections were treated with commercial biotinylated secondary anti-immunoglobulin, followed by avidin coupled to biotinylated horseradish peroxidase, at room temperature, according to the manufacturer's instructions (LSAB2 kit, DAKO Corporation). Immunohistochemical reactions were developed with diaminobenzidine as the chromogenic peroxidase substrate. Sections were counterstained with hematoxylin after immunohistochemistry. Specificity was verified by negative control reactions without primary antibody, as well as appropriate cytoplasmic reactions for each antigen in positive control tissues. For VEGF expression, immunohistochemical reactions were assessed in areas of invasive carcinoma and non-neoplastic breast tissue. Cytoplasmic staining intensity was graded from 0 (no staining) to 3 (most intensely stained), and the percentage of positive cells was noted.

\section{Microvessel Density Assessment}

\section{Direct microscopy}

Immunohistochemical reactions for CD31 and D2-40 antigen were interpreted independently by two authors (WWLC, CC) using a two-headed microscope. The two most vascularized areas within 
tumor ('hot spots') were chosen at low magnification $(\times 40)$ and vessels were counted in a representative high magnification $\left(\times 400 ; 0.152 \mathrm{~mm}^{2} ; 0.44 \mathrm{~mm}\right.$ diameter) field in each of these two areas. The high-magnification fields were then marked for subsequent image cytometric analysis. Single immunoreactive endothelial cells, or endothelial cell clusters separate from other microvessels, were counted as individual microvessels. Endothelial staining in large vessels with tunica media, and nonspecific staining of nonendothelial structures, were disregarded in microvessel counts. Mean visual microvessel density for CD31 and D2-40 was calculated as the average of four counts (two authors and two microscopic fields).

\section{Image cytometry}

Tissue sections stained with anti-CD31 and D2-40 were analyzed with the Ariol SL-50 ${ }^{\mathrm{TM}}$ system (Applied Imaging, Santa Clara, CA, USA). Ariol SL-50 ${ }^{\mathrm{TM}}$ is an automated scanning microscope controlled by a computer with software that directs a motorized stage, filter-wheels and automated focusing system. The Kisight image analysis application was used to quantify microvessel density. The default Kisight assay executes three passes (at $\times 12.5, \times 50$ and $\times 200$ total magnifications). The initial low-magnification pass locates tissue on the slide and creates digital scans for the pass at intermediate magnification. Automated image analysis at this magnification identifies brown-stained ring-like structures based on color and morphometry. Areas marked during direct microscopic microvessel counting were selected on intermediate magnification images, using drawing tools for reprocessing. Microvessel density was calculated as the percentage of total field area positively stained for vascular marker.

\section{Quantitative RT-PCR for VEGF-Family Gene Products}

Reactions were performed on formalin-fixed paraffin-embedded tissues using published protocols with minor modifications. ${ }^{18}$ Histologic sections were deparaffinized with xylene and rehydrated with ethanol. Areas containing tumor were microdissected with a sterile scalpel into digestion buffer. Samples were heated at $95^{\circ} \mathrm{C}$ for $10 \mathrm{~min}$ and digested with proteinase $\mathrm{K}$ at $52^{\circ} \mathrm{C}$ for $4 \mathrm{~h}$. Total RNA was isolated by phenol/chloroform extraction followed by ethanol precipitation. Quantitative RT-PCR assays were performed using standard SYBR-Green methodology on the I-cycler system (Bio-Rad). Intron-spanning PCR primers for the VEGF, VEGF-C and VEGF-D were designed with Primer Express software (Applied Biosystems) according to the manufacturer's instructions. Primers and amplicon sizes were as follows. VEGF: forward 5'-GCTGTCTTGGGTGCATTGG-3'; reverse 5'-GCAGCCTGGGACCACTTG-3'; Amplicon size
69 bp. VEGF-C: forward 5'-CAGTGTCAGGCAGC GAACA-3'; reverse 5'-TTCCTGAGCCAGGCATCTG$3^{\prime}$; amplicon size $78 \mathrm{bp}$. VEGF-D: forward $5^{\prime}$ TGGGATAGCAACAAATGTAAATGTG-3'; reverse 5'-GCAACGATCTTCGTCAAACATC-3'; amplicon size $126 \mathrm{bp}$. Quantification was based on cycle number required to reach an SYBR-Green fluorescence threshold in the linear phase of amplification. Reaction specificity was assessed by comparing amplicon melting points and size with values predicted by amplicon sequences. Expression levels of VEGF, VEGF-C and VEGF-D were normalized to $28 \mathrm{~S}$ ribosomal RNA. For all cases, total RNA was prepared from two independent, replicate sections to assess interassay variability. Interassay coefficients of variation were generally less than 10\% (not shown).

\section{Statistical Analyses}

Three levels of statistics were performed using the SAS version 8.2 (Copyright ${ }^{\odot}$ 1999-2001 by SAS Institute Inc., Cary, NC, USA): (a) Pearson's correlations; (b) $2 \times 2 \chi^{2}$ and Fisher's exact tests; and (c) Kappa agreement tests. Pearson's correlations were validated using graphical methods such as scattergrams. Specifically, the relationship between mean manual CD31 and D2-40 microvessel density with their mean image cytometry counterparts, the relationship between VEGF immunohistochemical results and mean CD31 and D2-40 values (both manual and image cytometry), the relationship between VEGF family protein PCR results and mean CD31 and D2-40 values (both manual and image cytometry), and the relationship between clinicopathologic factors and mean CD31 and D2-40 values (both manual and image cytometry) were all analyzed by Pearson's correlation coefficients. Only Pearson's correlation coefficients with an accompanying $P$-value of $<0.05$ were considered statistically significant. $\chi^{2}$ and Fisher's exact tests were used to compare VEGF, VEGF-C and VEGF-D (both immunohistochemical results and PCR results) with microvessel density and clinicopathologic parameters.

\section{Results}

Clinicopathologic parameters of the 29 invasive breast carcinoma studied are summarized as follows. Median patient age was 66 years (range 34-91 years). Histologic diagnoses included ductal $(N=22)$, lobular $(N=6)$ and papillary $(N=1)$ carcinomas. Histologic grades included grade I $(N=7)$, grade II $(N=16)$ and grade III $(N=6)$. Tumor stages included pT1 $(N=1)$, pT2 $(N=9)$, pT3 $(N=1)$ and pT4 $(N=1)$. Status of regional lymph nodes, hormone receptors and HER2/neu were: lymph node metastasis positive $(N=14)$ and negative $(N=15)$, estrogen receptor positive $(N=23)$ and 
negative $(N=6)$, progesterone receptor positive $(N=14)$ and negative $(N=15)$, and HER2/neu positive $(N=15)$ and negative $(N=14)$. Case characterization is compiled in Table 1.

\section{Correlation of Microvessel Density with Clinicopathologic Parameters of Breast Cancer}

Angiogenesis and lymphangiogenesis were quantified by visual and image cytometric microvessel density, using CD31 and D2-40 as markers for vascular and lymphatic endothelium, respectively
(Figures 1 and 2). For CD31, mean microvessel density was 29.2 microvessels per $\times 200$ field visually (range $12.8-94.3$ ), or $2.4 \%$ area per $\times 200$ field by image cytometry (range $0.0-8.2$ ). For D2-40, mean microvessel density was 7.4 lymphatic microvessels per $\times 200$ field visually (range $0.0-24.0$ ), or $2.1 \%$ area per $\times 200$ field by image cytometry (range 0.0-8.2). Table 2 shows Pearson's correlations between visual and image cytometric microvessel density for CD31 and D2-40. CD31 microvessel density showed similar trends using either technique, but the correlation was not statistically significant $(r=0.2779 ; P=0.1521)$. In contrast, visual

Table 1 Clinicopathologic features of the specimen cohort

\begin{tabular}{|c|c|c|c|c|c|c|c|c|}
\hline Patients & Age (years) & Histology & Primary tumor size $(\mathrm{cm}) /$ stage $\mathrm{a}^{\mathrm{a}}$ & Grade & $L N$ & $E R$ & $P R$ & Her2/neu \\
\hline 1 & 53 & Ductal & $5.5 / \mathrm{pT} 3$ & 3 & POS & NEG & POS & NEG \\
\hline 2 & 72 & Ductal & $0.9 / \mathrm{pT} 1 \mathrm{~b}$ & 3 & POS & POS & POS & POS \\
\hline 3 & 78 & Ductal & $2.7 / \mathrm{pT} 2$ & 3 & POS & NEG & NEG & NEG \\
\hline 4 & 50 & Ductal & $2.3 / \mathrm{pT} 2$ & 2 & NEG & POS & POS & POS \\
\hline 5 & 37 & Ductal & $1.7 / \mathrm{pT} 1 \mathrm{c}$ & 2 & POS & POS & POS & NEG \\
\hline 6 & 73 & Ductal & $0.5 / \mathrm{pT} 1 \mathrm{a}$ & 1 & NEG & POS & POS & POS \\
\hline 7 & 66 & Ductal & $1.2 / \mathrm{pT} 1 \mathrm{c}$ & 3 & NEG & POS & NEG & POS \\
\hline 8 & 66 & Papillary & 1/pT1b & 1 & NEG & POS & POS & NEG \\
\hline 9 & 58 & Ductal & $0.8 / \mathrm{pT} 1 \mathrm{~b}$ & 1 & POS & POS & POS & NEG \\
\hline 10 & 51 & Lobular & $1.3 / \mathrm{pT} 1 \mathrm{c}$ & 2 & NEG & POS & POS & NEG \\
\hline 11 & 75 & Ductal & $0.3 / \mathrm{pT} 1 \mathrm{a}$ & 2 & POS & POS & POS & NEG \\
\hline 12 & 55 & Lobular (L\&R) & 1.1(L:0.9, R:1.1)/pT1c & 2 & POS & POS & NEG & POS \\
\hline 13 & 66 & Ductal & $1.4 / \mathrm{pT} 1 \mathrm{c}$ & 1 & NEG & POS & NEG & NEG \\
\hline 14 & 70 & Ductal & $0.3 / \mathrm{pT} 1 \mathrm{a}$ & 2 & POS & NEG & NEG & NEG \\
\hline 15 & 86 & Ductal & $2.9 / \mathrm{pT} 2$ & 2 & NEG & NEG & NEG & POS \\
\hline 16 & 58 & Lobular & $1.5 / \mathrm{pT} 1 \mathrm{c}$ & 2 & NEG & POS & NEG & POS \\
\hline 17 & 43 & Ductal & 0.9/pT1b & 2 & NEG & POS & NEG & NEG \\
\hline 18 & 46 & Ductal & $1.2 / \mathrm{pT} 1 \mathrm{c}$ & 1 & NEG & POS & POS & NEG \\
\hline 19 & 67 & Ductal & $4.6 / \mathrm{pT} 2$ & 2 & NEG & NEG & NEG & POS \\
\hline 20 & 52 & Ductal & $1.8 / \mathrm{pT} 1 \mathrm{c}$ & 3 & NEG & NEG & NEG & POS \\
\hline 21 & 67 & Ductal & $2.3 / \mathrm{pT} 2$ & 2 & NEG & POS & POS & POS \\
\hline 22 & 66 & Lobular & $2.7 / \mathrm{pT} 2$ & 2 & POS & POS & NEG & POS \\
\hline 23 & 70 & Ductal & 2/pT1c & 2 & POS & POS & NEG & POS \\
\hline 24 & 34 & Ductal & $2.4 / \mathrm{pT} 2$ & 2 & POS & POS & POS & POS \\
\hline 25 & 71 & Lobular & $2.2 / \mathrm{pT} 2$ & 2 & POS & POS & POS & POS \\
\hline 26 & 69 & Ductal & 1.1pT1c & 1 & NEG & POS & POS & NEG \\
\hline 27 & 56 & Lobular & $2.3 / \mathrm{pT} 2$ & 2 & POS & POS & NEG & NEG \\
\hline 28 & 53 & Ductal & $0.2 / \mathrm{pT} 1 \mathrm{a}$ & 1 & NEG & POS & NEG & NEG \\
\hline 29 & 91 & Ductal & 10/pT4 & 3 & POS & POS & NEG & POS \\
\hline
\end{tabular}

$\mathrm{LN}=$ lymph node status; $\mathrm{ER}=$ estrogen receptor status; $\mathrm{PR}=$ progesterone receptor status; $\mathrm{POS}=$ positive; $\mathrm{NEG}=$ negative.

${ }^{\text {a }}$ Primary tumor stage according to AJCC 2002; insufficient patient data for stage grouping.

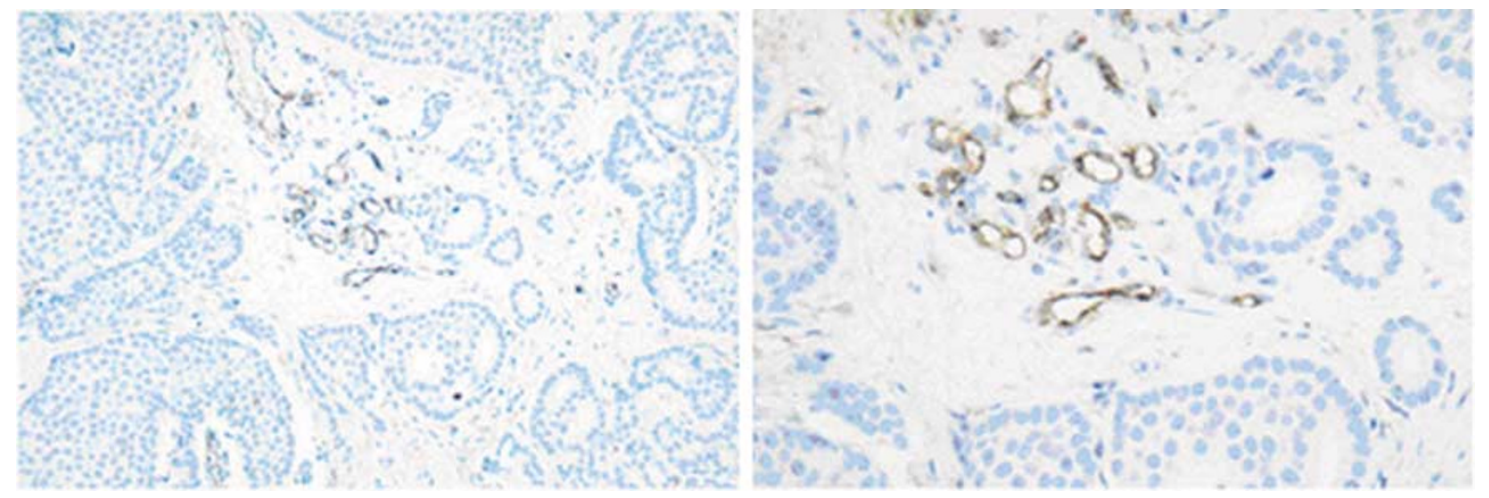

Figure 1 CD31 immunostained blood vessels in an invasive breast carcinoma 'hot spot', quantified visually (mean microvessel density $=54$ ) and image cytometrically (mean microvessel density $=4.0 \%$ area), $\times 200$ (left) and $\times 400$ (right). 


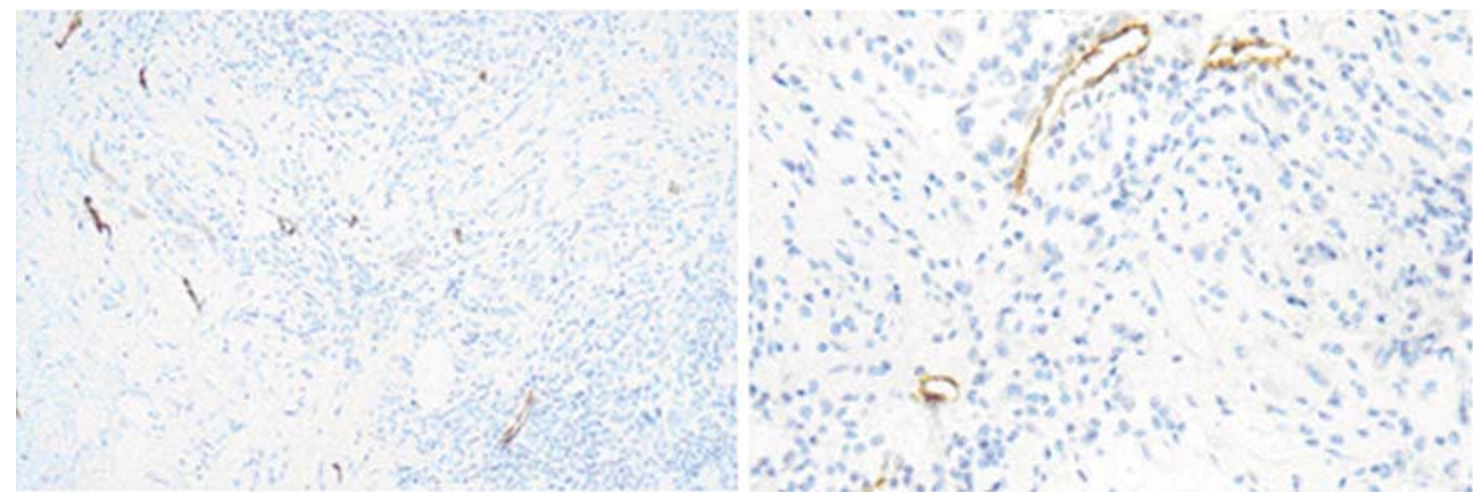

Figure 2 D2-40 immunostained lymphatics in an invasive breast carcinoma 'hot spot', quantified visually (mean microvessel density $=24$ ) and image cytometrically (mean microvessel density $=5.5 \%$ area), $\times 200$ (left) and $\times 400$ (right).

Table 2 Comparison of CD31 and D2-40 mean microvessel density assessed visually and by image cytometry (expressed as Pearson's correlations with associated $P$-values)

\begin{tabular}{lcccc}
\hline & CD31 (visual) & $D 2-40$ (visual) & CD31 (image cytometric) & D2-40 (image cytometric) \\
\hline $\begin{array}{l}\text { CD31 } \\
\text { (visual) }\end{array}$ & 1 & 0.1157 & 0.2779 & 0.1539 \\
$\begin{array}{l}\text { D2-40 } \\
\text { (visual) }\end{array}$ & 0.1157 & $(P=0.5502)$ & $(P=0.1521)$ & $(P=0.4343)$ \\
CD31 & $(P=0.5502)$ & 0.5589 & 0.4503 \\
(image cytometric) & $(P=0.1521)$ & $(\boldsymbol{P}=\mathbf{0 . 0 0 2 0})$ & $(\boldsymbol{P}=\mathbf{0 . 0 0 2 0})$ & $\mathbf{0 . 0 1 6 2 )}$ \\
$\begin{array}{l}\text { D2-40 } \\
\text { (image cytometric) }\end{array}$ & 0.1538 & 0.4503 & 1 & $\mathbf{0 . 3 8 1 2}$ \\
& $(P=0.4343)$ & $(\boldsymbol{P}=\mathbf{0 . 0 1 6 2})$ & $\mathbf{0 . 0 4 9 8 )}$ & 1 \\
\end{tabular}

Significant correlations are in boldface.

and image cytometric measurements of D2-40positive vessels correlated strongly $(r=0.4503$; $P=0.0162$ ). D2-40 microvessel density (using either technique) correlated significantly with image cytometric, but not visual, CD31 microvessel density $(r=0.5589, P=0.0020$ for visual D2-40; $r=0.3812$, $P=0.0498$ for image cytometric D2-40).

Table 3 shows Pearson's correlations of CD31 and D2-40 microvessel density with clinicopathologic parameters of breast cancer. Mean image cytometric CD31 microvessel density correlated significantly with tumor size $(P=0.0001)$, histologic grade $(P=0.0107)$, lymph node status $(P=0.0395)$, and stage $(P=0.0035)$. Mean image cytometric D2-40 microvessel density correlated significantly with stage $(P=0.0123)$, while visual $\mathrm{D} 2-40$ analysis trended with lymph node metastasis $(P=0.0558)$. Angiogenesis and lymphangiogenesis did not correlate significantly with estrogen receptor, progesterone receptor or HER2/neu immunoreactivity.

\section{Correlation of Microvessel Density with VEGF, VEGF-C and VEGF-D Gene Expression}

VEGF was detected immunohistochemically in breast carcinoma and vascular endothelial cells (Figure 3). The correlations of CD31 and D2-40 microvessel density with immunohistochemical
VEGF expression are shown in Table 4. Tumor angiogenesis and lymphangiogenesis, determined as mean CD31 and D2-40 microvessel density, correlated significantly with VEGF expression in the endothelial cells of peritumoral benign breast tissue $(P=0.0217$ and 0.0012 , respectively, by image cytometry), but not with VEGF expression in carcinoma cells or intratumoral endothelial cells. VEGF antigen in peritumoral endothelial cells also correlated with pathologic stage pT2 or higher $\left(P=0.0025\right.$ and 0.0049 for $\chi^{2}$-test and Fisher's Exact Test). Table 5 shows the correlation of CD31 and D240 with VEGF, VEGF-C and VEGF-D mRNA expression by Q-RT-PCR. Visual mean D2-40 microvessel density correlated significantly with VEGF-C and VEGF-D mRNA $(P=0.0291$ and 0.0432 , respectively), but not with VEGF. Although image cytometric D2-40 microvessel density also trended with VEGF-C and VEGF-D expression, the correlations did not achieve statistical significance. CD31 microvessel density did not correlate significantly with VEGF, VEGF-C or VEGF-D mRNA expression. In addition to their correlation with D2-40 microvessel density, VEGF-C and VEGF-D were associated with the histologic type of invasive ductal carcinoma $\left(P=0.0218\right.$ and 0.0469 , respectively, for $\chi^{2}$-test), and VEGF-C mRNA correlated significantly with lymph node status $\left(P=0.0221\right.$ and 0.0414 for $\chi^{2}$-test and Fisher's exact test). 
Table 3 Correlations of mean CD31 and D2-40 microvessel density, visually and image cytometrically, with clinicopathologic parameters of breast carcinoma (expressed as Pearson's correlations with associated $P$-values)

\begin{tabular}{|c|c|c|c|c|}
\hline \multirow[t]{2}{*}{ Clinicopathologic parameter } & \multicolumn{4}{|c|}{ Microvessel density technique } \\
\hline & $\begin{array}{c}C D 31 \\
\text { (visual) }\end{array}$ & $\begin{array}{c}\text { D2-40 } \\
\text { (visual) }\end{array}$ & $\begin{array}{c}C D 31 \\
\text { (image cytometric) }\end{array}$ & $\begin{array}{c}D 2-40 \\
\text { (image cytometric) }\end{array}$ \\
\hline Patient age & $\begin{array}{c}-0.0878 \\
(P=0.6507)\end{array}$ & $\begin{array}{c}-0.3074 \\
(P=0.1048)\end{array}$ & $\begin{array}{c}0.1062 \\
(P=0.5907)\end{array}$ & $\begin{array}{c}-0.3553 \\
(P=0.0635)\end{array}$ \\
\hline Tumor size & $\begin{array}{c}0.2998 \\
(P=0.1141)\end{array}$ & $\begin{array}{c}0.1213 \\
(P=0.5308)\end{array}$ & $\begin{array}{c}0.6669 \\
(\boldsymbol{P}=\mathbf{0 . 0 0 0 1})\end{array}$ & $\begin{array}{c}0.0879 \\
(P=0.6567)\end{array}$ \\
\hline Histologic type & $\begin{array}{c}-0.0039 \\
(P=9841)\end{array}$ & $\begin{array}{c}-0.1418 \\
(P=0.4633)\end{array}$ & $\begin{array}{c}0.1255 \\
(P=0.5247)\end{array}$ & $\begin{array}{c}-0.0501 \\
(P=0.8001)\end{array}$ \\
\hline Histologic grade & $\begin{array}{c}-0.0912 \\
(P=0.6382)\end{array}$ & $\begin{array}{c}0.0360 \\
(P=0.8531)\end{array}$ & $\begin{array}{c}0.4746 \\
(P=\mathbf{0 . 0 1 0 7})\end{array}$ & $\begin{array}{c}-0.0077 \\
(P=0.9690)\end{array}$ \\
\hline Lymph node status & $\begin{array}{c}-0.1840 \\
(P=0.3393)\end{array}$ & $\begin{array}{c}0.3590 \\
(\boldsymbol{P}=\mathbf{0 . 0 5 5 8})\end{array}$ & $\left(\begin{array}{c}0.3912 \\
(\boldsymbol{P}=\mathbf{0 . 0 3 9 5})\end{array}\right.$ & $\begin{array}{c}0.1713 \\
(P=0.3835)\end{array}$ \\
\hline Tumor stage & $\begin{array}{c}0.3529 \\
(P=0.0604)\end{array}$ & $\begin{array}{c}0.2632 \\
(P=0.1677)\end{array}$ & $\begin{array}{c}0.5324 \\
(\boldsymbol{P}=\mathbf{0 . 0 0 3 5})\end{array}$ & $\begin{array}{c}0.4669 \\
(\boldsymbol{P}=\mathbf{0 . 0 1 2 3})\end{array}$ \\
\hline Estrogen receptor status & $\begin{array}{c}-0.2516 \\
(P=0.1880)\end{array}$ & $\begin{array}{c}-0.0435 \\
(P=0.8227)\end{array}$ & $\begin{array}{c}-0.3425 \\
(P=0.0744)\end{array}$ & $\begin{array}{c}0.0442 \\
(P=0.8230)\end{array}$ \\
\hline Progesterone receptor status & $\begin{array}{c}-0.1886 \\
(P=0.3272)\end{array}$ & $\begin{array}{c}0.0650 \\
(P=0.7378)\end{array}$ & $\begin{array}{c}-0.0553 \\
(P=0.7799)\end{array}$ & $\begin{array}{c}0.3430 \\
(P=0.0739)\end{array}$ \\
\hline HER2/neu status & $\begin{array}{c}0.1576 \\
(P=0.4141)\end{array}$ & $\begin{array}{c}-0.1660 \\
(P=0.3894)\end{array}$ & $\begin{array}{c}-0.0260 \\
(P=0.8957)\end{array}$ & $\begin{array}{c}0.0152 \\
(P=0.9389)\end{array}$ \\
\hline
\end{tabular}

Significant correlations are in boldface.

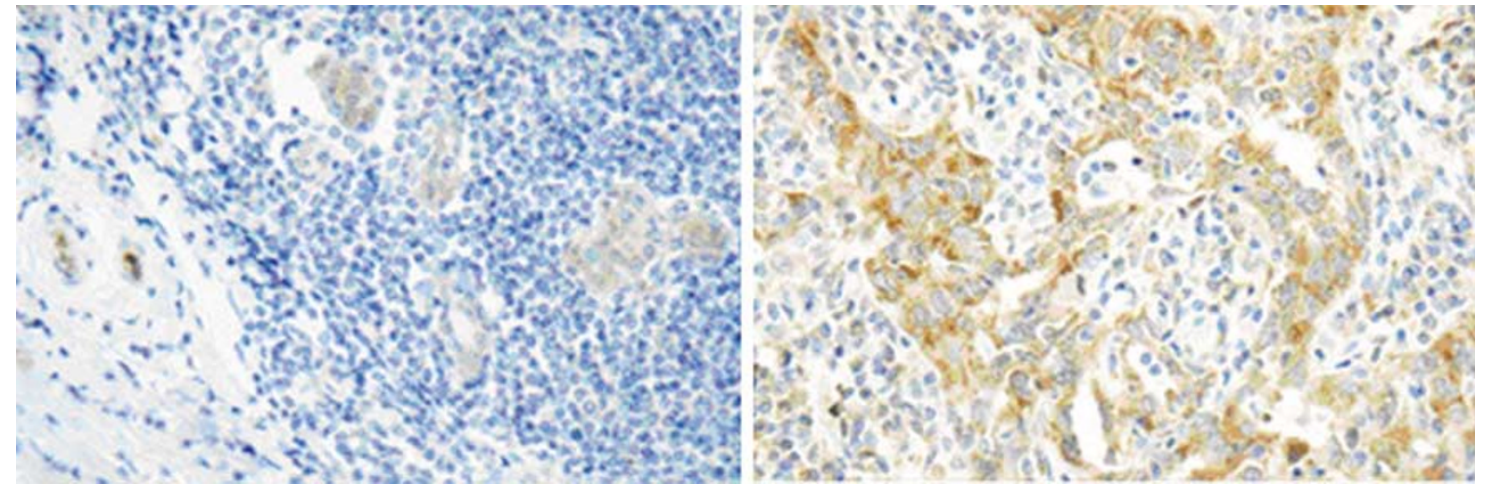

Figure 3 VEGF protein expression in blood vessels in normal breast tissue (left, $\times 400)$ and in breast carcinoma cells $($ right, $\times 400)$

\section{Discussion}

This study is one of the first attempts to quantify breast cancer angiogenesis and lymphangiogenesis concurrently by microvessel density using CD31 and the novel lymphatic marker D2-40. We compared the performance of visual $v s$ automated microvessel density for CD31 and D2-40, and related these measurements with clinicopathologic parameters of breast cancer. In addition, to explore mechanisms of angiogenesis and lymphangiogenesis in clinical breast cancer specimens, we compared CD31 and D2-40 microvessel density with VEGF, VEGF-C and VEGF-D expression by immunohistochemistry and fixed-tissue quantitative RT-PCR. Blood and vascular microvessel density correlated with prognostic parameters including lymph node metastasis, while VEGF family gene expression correlated with microvessel density. Thus, measurements of angiogenesis and lymphangiogenesis may have clinical utility in the evaluation of breast cancer, particularly for estimation of metastatic risk, if our findings are confirmed in prospective studies. This suggestion is supported by several studies showing microvessel density to be a significant prognostic marker for breast cancer. For example, a recent Japanese study showed that microvessel density, determined visually with anti-Factor VIII-related antigen, was an independent predictor of disease-free and overall survival in a series of 252 cases. ${ }^{19}$ A meta-analysis of 43 independent studies determined that high microvessel density was associated significantly with breast cancer survival, particularly in node-negative cases. ${ }^{4}$ However, the relative risks defined in this meta-analysis were too small to make unequivocal conclusions on the clinical utility of microvessel counting. Differences in microvessel counting techniques and targeted endothelial antigens were 
Table 4 Comparisons of mean CD31 and D2-40 microvessel density, visually and image cytometrically, with immunohistochemical expression of vascular endothelial growth factor (expressed as Pearson's correlations with associated $P$-values)

VEGF expression

Microvessel density technique

\begin{tabular}{|c|c|c|c|c|}
\hline & \\
\hline & $\begin{array}{c}C D 31 \\
\text { (visual) }\end{array}$ & $\begin{array}{c}\text { D2-40 } \\
\text { (visual) }\end{array}$ & $\begin{array}{c}\text { CD31 } \\
\text { (image cytometric) }\end{array}$ & $\begin{array}{c}D 2-40 \\
\text { (image cytometric) }\end{array}$ \\
\hline Malignant cells & 0.235 & -0.29 & -0.1081 & 0.1517 \\
\hline & $(P=0.2204)$ & $(P=0.1333)$ & $(P=0.5841)$ & $(P=0.4410)$ \\
\hline Intratumoral vascular endothelium & $\begin{array}{c}0.275 \\
\end{array}$ & $\begin{array}{l}0.07 \\
(P-07081)\end{array}$ & 0.1634 & $\begin{array}{c}0.1914 \\
(P=03390)\end{array}$ \\
\hline Peritumoral vascular endothelium & $\begin{array}{c}(P=0.1574) \\
0.493 \\
(P=\mathbf{0 . 0 1 7 0})\end{array}$ & $\begin{array}{c}(P=0.7081) \\
0.35 \\
(P=0.1060)\end{array}$ & $\begin{array}{c}(P=0.4154) \\
0.4864 \\
(P=\mathbf{0 . 0 2 1 7})\end{array}$ & $\begin{array}{c}(P=0.3390) \\
0.6325 \\
(\boldsymbol{P}=\mathbf{0 . 0 0 1 2})\end{array}$ \\
\hline
\end{tabular}

Significant correlations are in boldface.

VEGF $=$ vascular endothelial growth factor.

Table 5 Correlation of mean CD31 and D2-40 microvessel density with $\log _{2}$ relative expression of VEGF, VEGF-C and VEGF-D by quantitative RT-PCR (expressed as Pearson's correlations with corresponding $P$-values)

\begin{tabular}{|c|c|c|c|c|}
\hline \multirow{2}{*}{$\begin{array}{l}\text { Gene } \\
\text { product }\end{array}$} & \multicolumn{4}{|c|}{ Microvessel density technique } \\
\hline & $\begin{array}{c}C D 31 \\
\text { (visual) }\end{array}$ & $\begin{array}{c}\text { D2-40 } \\
\text { (visual) }\end{array}$ & $\begin{array}{c}\text { CD31 } \\
\text { (image } \\
\text { cytometric) }\end{array}$ & $\begin{array}{c}\text { D2-40 } \\
\text { (image } \\
\text { cytometric) }\end{array}$ \\
\hline VEGF & $\begin{array}{c}-0.1126 \\
(P=0.5921)\end{array}$ & $\begin{array}{c}0.0171 \\
(P=0.9352)\end{array}$ & $\begin{array}{c}-0.0758 \\
(P=0.7247)\end{array}$ & $\begin{array}{c}-0.2029 \\
(P=0.3307)\end{array}$ \\
\hline VEGF-C & $\begin{array}{c}-0.0388 \\
(P=0.8539)\end{array}$ & $\begin{array}{c}0.4365 \\
(\boldsymbol{P}=\mathbf{0 . 0 2 9 1})\end{array}$ & $\begin{array}{c}0.2772 \\
(P=0.1898)\end{array}$ & $\begin{array}{c}0.3030 \\
(P=0.1409)\end{array}$ \\
\hline VEGF-D & $\begin{array}{c}-0.0020 \\
(P=0.9923)\end{array}$ & $\begin{array}{c}0.4075 \\
(\boldsymbol{P}=\mathbf{0 . 0 4 3 2})\end{array}$ & $\begin{array}{c}0.2629 \\
(P=0.2145)\end{array}$ & $\begin{array}{c}0.2938 \\
(P=0.1540)\end{array}$ \\
\hline
\end{tabular}

Significant correlations are in boldface.

VEGF = vascular endothelial growth factor; RT-PCR = reverse transcriptase-polymerase chain reaction.

considered likely sources of variability among the analyzed studies. Furthermore, several studies have shown no significant relationship between microvessel density and clinicopathologic parameters of breast cancer. For example, Axelsson et al assessed angiogenesis visually with anti-Factor VIII-associated antigen and found no correlation with lymph node status or any other breast cancer parameter. A major factor limiting the strength of association was high interobserver variability in microvessel counting. ${ }^{20}$ In a large Italian study, a significant correlation was shown between microvessel density (assessed visually with anti-CD34) and Her2/neu overexpression, but not lymph node metastasis or patient survival. ${ }^{21}$ Among node negative cases, this study focused on tumors negative for estrogen and progesterone receptors, which is distinct from the cohort in our research. Overall, the clinical significance of high microvessel density in breast cancer remains uncertain, and the variability in technical approaches and difficulty in distinguishing blood and lymphatic microvessels appears to contribute to this uncertainty.

Research on tumor lymphangiogenesis has lagged behind that of angiogenesis. Indeed, the existence of intratumoral lymphatic channels in breast cancer remains a controversial topic. ${ }^{9,22}$ A major impediment to the study of lymphangiogenesis in solid tumor growth and spread was the lack of a specific lymphatic marker. Several candidate lymphatic endothelial markers have been established recently, including the integral membrane glycoprotein podoplanin; ${ }^{23,24}$ the desmosomal component desmoplakin; ${ }^{25}$ the transcription factor Prox $1 ;^{26}$ the transmembrane 4 superfamily protein CD9;27 the receptor for VEGF-C and VEGF-D (VEGFR-3); and the LYVE-1 antigen. ${ }^{9,10}$ However, while each marker appears to be expressed preferentially in lymphatic endothelium, expression in other cell types has been described. Of particular relevance to the current study, VEGFR-3 and LYVE-1 may be expressed in certain blood vessel endothelia, complicating their use in differentiating lymphangiogenesis from angiogenesis. ${ }^{28}$

D2-40 antibody was recently reported to detect a fixation-resistant epitope on a $40 \mathrm{kDa}$ O-linked sialoglycoprotein expressed in lymphatic endothelium but not blood vessels. ${ }^{6}$ D2-40 was shown to immunostain lymphangiomas (benign tumors of undisputed lymphatic origin), but not benign neoplasms or tumor-like lesions of blood vessel origin. ${ }^{6}$ Although D2-40 reacted with malignant endothelial tumors with features of blood vessels, including angiosarcomas and Kaposi sarcomas, these tumors have been shown to coexpress lymphatic markers such as podoplanin and VEGFR-3 in a significant proportion of cases. ${ }^{29-33}$ In the setting of breast cancer, in which the associated blood and lymphatic vessels are nonmalignant, D2-40 immunoreactivity appears to be specific for lymphatic phenotype, making D2-40 an appropriate marker to assess lymphangiogenesis specifically.

Visual and image cytometric microvessel density counting methods are each associated with key advantages and limitations. For example, microscopic visual counting is less expensive and much more widely available among pathologists, but the inherent subjectivity of this method may limit interobserver reproducibility. ${ }^{20}$ In contrast, image 
cytometry is likely to be more objective and reproducible and can measure several unique morphometric parameters. Apart from the number of vessels within a predetermined area, many image cytometry software packages can also measure vessel luminal area, vessel luminal perimeter and the number of immunostained areas per microscopic field or scanned area. ${ }^{34,35}$ In the past few years, image cytometric microvessel area and microvessel perimeter have been demonstrated as independent prognostic factors in invasive ductal carcinoma. ${ }^{36,37}$

In light of the frequent use of both visual and image cytometric microvessel density counting methods, a question that continues to arise is how well data from the two approaches correlate. In our study, while visual and image cytometric CD31 microvessel density gave similar data, direct correlation did not reach statistical significance, and only image cytometric data were associated significantly with clinicopathologic parameters of breast cancer. In contrast, direct correlation of visual and image cytometric D2-40 microvessel density was statistically significant in our study. Nevertheless, tumor stage correlated only with image cytometric lymphangiogenesis, while lymph node status and VEGF family gene expression correlated only with visual D2-40 data. Factors limiting the strength of correlations in our study may include the subjective nature of visual estimates, the confounding signals from nonendothelial structures (eg stromal connective tissues or plasma cells, which may react with CD31 and be counted by the image cytometer but not by experienced pathologists), and the study size. Previous reports in the literature also show conflicting correlation between visual and image cytometric analysis of vessel counts in breast carcinomas. When anti-CD31 was used for vascular staining, Barbareschi et $a l^{38}$ demonstrated a statistically significant correlation between visual and computer image analysis microvessel counts in 91 breast carcinoma cases. The statistical power of this study may be accounted for by the greater case number than that of our study. Differences in methodologies between our study and Barbareschi's study might also play a role. For example, microvessels within tumors were counted in their study. This also reflects part of the subjective nature of the microvessel counting process. Fox et $a l^{39}$ also used antiCD31 as the tool for angiogenesis research and reported a statistically significant correlation between various vascular parameters (luminal area, luminal perimeter and microvessel number) with a subjective, visual three-tier vascular grading system under the microscope. While this is an innovative method of estimating angiogenesis, it is difficult to compare its results with our and other studies using more common methods of microvessel counting. In contrast to the aforementioned studies, Kohlberger et $a l,{ }^{40}$ using anti-factor VIII to assess angiogenesis, reported no significant correlation between manual microvessel counting and computer image analysis.
Thus, to summarize previous studies correlating visual and computer image analysis microvessel density, diversity exists in the degrees of correlation reported, likely related to differences in target vascular antigens, methods used for microvessel counting, sample sizes and possibly other factors.

Assays that probe molecular mechanisms of tumor angiogenesis and lymphangiogenesis have potential to increase the clinical utility of microvessel density studies, since they may allow the observer to distinguish varying signal transduction pathways with distinct prognostic or therapeutic implications. For example, the advent of specific VEGF inhibitors for antiangiogenic therapy makes it important to determine if expression of VEGF-family gene products correlates consistently with microvessel density. ${ }^{41}$ To explore the relationship of vascular and lymphatic microvessel density with VEGF, VEGF-C and VEGF-D expression, we developed immunohistochemical and quantitative RT-PCR assays for these growth factors that utilize formalin-fixed paraffinembedded tissues, which are more generally accessible in routine diagnostic pathology settings. Immunohistochemical assays for all three growth factors have been described. ${ }^{42,43}$ However, most studies of VEGF-C and VEGF-D in cancer have employed RT-PCR, ${ }^{44,45}$ and we have only established reliable immunohistochemical assays for VEGF to date. Our data suggest that VEGF protein expression in blood vessels of benign tissue surrounding breast tumors correlates significantly with tumor angiogenesis and lymphangiogenesis determined by microvessel density. Similar relationships were not seen using quantitative RT-PCR, which may be due to the inability to localize the VEGF mRNA signals to specific cells.

Our ability to assay VEGF, VEGF-C and VEGF-D mRNA in formalin-fixed paraffin-embedded breast specimens is consistent with several recent studies, which have used fluorogenic quantitative RT-PCR to measure gene transcription in fixed tissues. ${ }^{46-49}$ Fluorogenic quantitative RT-PCR is designed to amplify very small sequences (200 bp or less), which can be extracted intact from fixed tissue with adequate efficiency for comparison among specimens. Assays for VEGF, VEGF-C and VEGF-D have potential clinical utility, based on growing evidence that these growth factors play important roles in lymphangiogenesis and lymph node metastasis in breast and other cancers. ${ }^{50,51}$ In our study, mRNA for VEGF-C and VEGF-D tended to correlate with D2-40 microvessel density and lymph node metastasis. Similarly, Skobe et al ${ }^{9}$ showed that VEGF-C overexpression in breast cancer cells increased intratumoral lymphangiogenesis, assessed by LYVE-1, and enhanced metastasis to regional lymph nodes and lungs. Mattila et $a l^{13}$ similarly demonstrated that VEGF-C overexpression stimulated tumor lymphangiogenesis and increased lymph node metastasis of the estrogen-dependent MCF-7 breast cancer cell line in a nude mice model. Stacker et $a l^{10}$ showed 
that VEGF-D, but not VEGF, promoted the metastatic spread of tumor cells via the lymphatics. Studies performed on other cancer types have yielded comparable results. Thus, Kitadai et al ${ }^{50}$ illustrated an association between VEGF-C expression in human esophageal squamous cell carcinomas with depth of tumor invasion, tumor stage, venous invasion, lymphatic invasion and lymph node metastasis. Likewise, both microvessel density and VEGF expression were significantly increased in nasopharyngeal carcinoma tissue with metastasis as compared with those without. ${ }^{51}$

To summarize, we used the recently described lymphatic endothelial immunomarker D2-40 to estimate lymphatic microvessel density in fixed breast tissues, and achieved comparable results by visual microscopy and image cytometry. In contrast, blood vascular microvessel density measurements with CD31 did not correlate using visual microscopy vs image cytometry. Microvessel density assays using CD31 and D2-40, and molecular assays for VEGF family gene expression, illustrated relationships of angiogenesis and lymphangiogenesis with tumor histology, grade, stage and lymph node metastasis. Based on these findings, prospective studies may be useful to determine if assays for blood and lymphatic microvessel density and VEGF family gene expression have clinical utility in defining risk for nodal metastasis in breast cancer.

\section{Acknowledgements}

We thank Avon Foundation Breast Cancer Research and Care Program, Winship Cancer Institute of Emory University, for monetary support, Applied Imaging Corporation (Santa Clara, CA, USA) for use of the Ariol SL-50 ${ }^{\mathrm{TM}}$ image analyzer and excellent technical support, Robert Santoianni for photographic expertise, and Judy Dunbar for secretarial assistance.

\section{Duality of interest}

The authors of this article declare no duality of interest.

\section{References}

1 Nathanson SD. Insights into the mechanisms of lymph node metastasis. Cancer 2003;98:413-423.

2 Locopo N, Fanelli M, Gasparini G. Clinical significance of angiogenic factors in breast cancer. Breast Cancer Res Treat 1998;52:159-173.

3 Folkman J. Angiogenesis and angiogenesis inhibition: an overview. Experientia Suppl 1997;79:1-8.

4 Uzzan B, Nicolas P, Cucherat M, et al. Microvessel density as a prognostic factor in women with breast cancer: a systematic review of the literature and metaanalysis. Cancer Res 2004;64:2941-2955.
5 Kahn HJ, Marks A. A new monoclonal antibody, D2-40, for detection of lymphatic invasion in primary tumors. Lab Invest 2002;82:1255-1257.

6 Kahn HJ, Bailey P, Marks A. Monoclonal antibody D2-40, a new marker of lymphatic endothelium, reacts with Kaposi's sarcoma and a subset of angiosarcomas. Mod Pathol 2002;15:434-440.

7 Tille JC, Nisato R, Pepper MS. Lymphangiogenesis and tumour metastasis. Novartis Found Symp 2004;256: 112-131.

8 Nisato RE, Tille JC, Pepper MS. Lymphangiogenesis and tumor metastasis. Thromb Haemost 2003;90: 591-597.

9 Skobe M, Hawighorsi T, Jackson DG, et al. Induction of tumor lymphangiogenesis by VEGF-C promotes breast cancer metastasis. Nat Med 2001;7:192-198.

10 Stacker SA, Caesar C, Baldwin ME, et al. VEGF-D promotes the metastatic spread of tumor cells via the lymphatics. Nat Med 2001;7:186-191.

11 Salven P, Lymboussaki A, Heikkila P, et al. Vascular endothelial growth factors VEGF-B and VEGF-C are expressed in human tumors. Am J Pathol 1998;153: 103-108.

12 Witmer AN, van Blijswijk BC, Dai J, et al. VEGFR-3 in adult angiogenesis. J Pathol 2001;195:490-497.

13 Mattila MM-T, Ruohola JK, Karpanen T, et al. VEGF-C induced lymphangiogenesis is associated with lymph node metastasis in orthotopic MCF-7 tumors. Int J Cancer 2002;98:946-951.

14 World Health Organization, Geneva, Switzerland. Histologic typing of breast tumors. Tumori 1982;68: 181-198.

15 Elston CE, Ellis IO. Pathological prognostic factors in breast cancer. I. The value of histologic grade in breast cancer: experience from a large study with long-term follow-up. Histopathology 1991;19:403-410.

16 Fitzgibbons PL, Page DL, Weaver D, et al. Prognostic factors in breast cancer. College of American Pathologists consensus statement 1999. Arch Pathol Lab Med 2000;124:966-978.

17 Norton AJ, Jordan S, Yeomans P. Brief, high-temperature heat denaturation (pressure cooking): a simple and effective method of antigen retrieval for routinely processed tissues. J Pathol 1994;173:371-379.

18 Specht K, Richter T, Muller U, et al. Quantitative gene expression analysis in microdissected archival formalin-fixed and paraffin-embedded tumor tissue. Am J Pathol 2001;158:419-429.

19 Tsutsui S, Kume M, Era S. Prognostic value of microvessel density in invasive ductal carcinoma of the breast. Breast Cancer 2003;10:312-319.

20 Axelsson K, Ljung BM, Moore II DH, et al. Tumor angiogenesis as a prognostic assay for invasive ductal breast carcinoma. J Natl Cancer Inst 1995;87:997-1008.

21 Ludovini V, Sidoni A, Pistola L, et al. Evaluation of the prognostic role of vascular endothelial growth factor and microvessel density in stages I and II breast cancer patients. Breast Cancer Res Treat 2003;81:159-168.

22 Williams CS, Leek RD, Robson AM, et al. Absence of lymphangiogenesis and intratumoural lymph vessels in human metastatic breast cancer. J Pathol 2003;200: 195-206.

23 Schoppmann SF, Birner P, Studer P, et al. Lymphatic microvessel density and lymphovascular invasion assessed by anti-podoplanin immunostaining in human breast cancer. Anticancer Res 2001;21: 2351-2355. 
24 Cursiefen C, Schlotzer-Schrehardt U, Kuchle M, et al. Lymphatic vessels in vascularized human corneas: immunohistochemical investigation using LYVE-1 and podoplanin. Invest Ophthalmol Vis Sci 2002;43: 2127-2135.

25 Ebata N, Nodasaka Y, Sawa Y, et al. Desmoplakin as a specific marker of lymphatic vessels. Microvasc Res 2001;61:40-48.

26 Wigle JT, Oliver G. Prox1 function is required for the development of the murine lymphatic system. Cell 1999;98:769-778.

27 Erovic BM, Neuchrist C, Kandutsch S, et al. CD9 expression on lymphatic vessels in head and neck mucosa. Mod Pathol 2003;16:1028-1034.

28 Stacker SA, Achen MG, Jussila L, et al. Lymphangiogenesis and cancer metastasis. Nat Rev Cancer 2002;2:573-583.

29 Breiteneder-Geleff S, Soleiman A, Kowalski H, et al. Angiosarcomas express mixed endothelial phenotypes of blood and lymphatic capillaries. Podoplanin as a specific marker for lymphatic endothelium. Am J Pathol 1999;154:385-394.

30 Folpe AL, Veikkola $\mathrm{T}$, Valtola $\mathrm{R}$, et al. Vascular endothelial growth factor receptor-3 (VEGFR-3): A marker of vascular tumors with presumed lymphatic differentiation, including Kaposi's sarcoma, Kaposiform and Dabska-type hemangioendotheliomas, and a subset of angiosarcoma. Mod Pathol 2000;13:180-185.

31 Weninger W, Partanen TA, Breiteneder-Geleff S, et al. Expression of vascular endothelial growth factor receptor-3 and podoplanin suggests a lymphatic endothelial cell origin of Kaposi's sarcoma tumor cells. Lab Invest 1999;79:243-251.

32 Jussila L, Valtola R, Partanen TA, et al. Lymphatic endothelium and Kaposi's sarcoma spindle cells detected by antibodies against the vascular endothelialgrowth factor receptor-3. Cancer Res 1998;58: 1599-1604.

33 Partanen TA, Alitalo K, Miettinen M. Lack of lymphatic vascular specificity of vascular endothelial growth factor receptor 3 in 185 vascular tumors. Cancer 1999;86:2406-2412.

34 Horak ER, Leek R, Klenk N, et al. Angiogenesis, assessed by platelet/endothelial cell adhesion molecule antibodies, as indicator of node metastases and survival in breast cancer. Lancet 1992;340:1120-1124.

35 Hasan J, Byers R, Jayson GC. Intra-tumoral microvessel density in human solid tumors. Br J Cancer 2002;86: 1566-1577.

36 Chantrain CF, DeClerck YA, Groshen S, et al. Computerized quantification of tissue vascularization using high-resolution slide scanning of whole tumor sections. J Histochem Cytochem 2003;51:151-158.

37 Olewniczak S, Chosia M, Kolodziej B, et al. Angiogenesis as determined by computerized image analysis and the risk of early relapse in women with invasive ductal breast carcinoma. Pol J Pathol 2003;54:53-59.

38 Barbareschi M, Weidner N, Gasparini G, et al. Microvessel density quantification in breast carcinomas.
Assessment by light microscopy versus a computer aided image analysis system. Appl Immunohistochem 1995;3:75-84.

39 Fox SB, Leek RD, Weekes MP, et al. Quantitation and prognostic value of breast cancer angiogenesis: comparison of microvessel density, Chalkley count, and computer image analysis. J Pathol 1995;177:275-283.

40 Kohlberger PD, Obermair A, Sliutz G, et al. Quantitative immunohistochemistry of factor VIII-related antigen in breast carcinoma: a comparison of computerassisted image analysis with established counting methods. Am J Clin Pathol 1996;105:705-710.

41 Pegram MD, Reese DM. Combined biological therapy of breast cancer using monoclonal antibodies directed against HER2/neu protein and vascular endothelial growth factor. Semin Oncol 2002;29:29-37.

42 Nakashima T, Kondoh S, Kitoh H, et al. Vascular endothelial growth factor-C expression in human gallbladder cancer and its relationship to lymph node metastasis. Int J Molec Med 2003;11:33-39.

43 White JD, Hewett PW, Kosuge D, et al. Vascular endothelial growth factor-D expression is an independent prognostic marker for survival in colorectal carcinoma. Cancer Res 2002;62:1669-1675.

44 Niki T, Iba S, Tokunou M, et al. Expression of vascular endothelial growth factors A, B, C, and D and their relationships to lymph node status in lung adenocarcinoma. Clin Cancer Res 2000;6:2431-2439.

45 Onogawa S, Kitadai Y, Tanaka S, et al. Expression of VEGF-C and VEGF-D at the invasive edge correlates with lymph node metastasis and prognosis of patients with colorectal carcinoma. Cancer Sci 2004;95: 32-39.

$46 \mathrm{Liu} \mathrm{H}$, Huang $\mathrm{X}$, Zhang $\mathrm{Y}$, et al. Archival fixed histologic and cytologic specimens including stained and unstained materials are amenable to RT-PCR. Diagn Mol Pathol 2002;11:222-227.

47 Coombs NJ, Gough AC, Primrose JN. Optimisation of DNA and RNA extraction from archival formalin-fixed tissue. Nucleic Acids Res 1999;27:e12.

48 Fritsch MK, Bridge JA, Schuster AE, et al. Performance characteristics of a reverse transcriptase-polymerase chain reaction assay for the detection of tumor-specific fusion transcripts from archival tissue. Pediatr Dev Pathol 2003;6:43-53.

49 Lassmann S, Bauer M, Soong R, et al. Quantification of CK20 gene and protein expression in colorectal cancer by RT-PCR and immunohistochemistry reveals inter- and intratumour heterogeneity. J Pathol 2002;198: 198-206.

50 Kitadai Y, Amioka T, Haruma K, et al. Clinicopathological significance of vascular endothelial growth factor (VEGF)-C in human esophageal squamous cell carcinomas. Int J Cancer 2001;93:662-666.

51 Guang-Wu H, Sungawa M, Jie-En L, et al. The relationship between microvessel density, the expression of vascular endothelial growth factor (VEGF), and the extension of nasopharyngeal carcinoma. Laryngoscope 2000;110:2066-2069. 